\title{
Phase-controlled two-qubit quantum gates
}

\author{
Vladimir S. Malinovsky \\ U.S. Army Research Laboratory, 2800 Powder Mill Road, Adelphi, Maryland 20783, USA \\ Ignacio R. Sola \\ Departamento de Quimica Fisica I, Universidad Complutense, 28040 Madrid, Spain \\ Jiri Vala \\ Department of Mathematical Physics, National University of Ireland, Maynooth, Ireland
}

(Received 20 January 2014; published 3 March 2014)

\begin{abstract}
We consider an implementation of a two-qubit entangling gate between trapped ions controlled by fully overlapping laser pulses. We particularly address the role of a relative phase between the pulses in the gate design. Though, in general, the relative phase determines the exact form of the two-qubit operation, it is shown that it affects only its single qubit part and has no effect on its entangling content. Based on a general canonical decomposition of the evolution operator we demonstrate that the controlled-NOT (CNOT) gate can be decomposed in terms of a two-qubit entangled gate and single qubit phase gates. Our study shows that any relative phase errors in two-qubit operations related to phase fluctuations of the external fields can be successfully corrected locally by single-qubit operations, which are usually easy to implement.
\end{abstract}

DOI: 10.1103/PhysRevA.89.032301

PACS number(s): 03.67.Lx, 03.67.Mn, 03.65.Ud, 42.50.Hz

\section{INTRODUCTION}

A universal set of quantum gates for quantum computation can be constructed from two single-qubit gates (the Hadamard gate and the phase gate) and a controlled-NOT (CNOT) twoqubit gate $[1,2]$. It is also proven that the CNOT gate can be replaced by a conditional two-qubit phase gate, which, in some cases, has easier experimental implementation. Single-qubit gates are simple rotations of the qubit and usually readily available for implementation in many different proposals for quantum computing as long as the Rabi oscillation between single-qubit states is demonstrated in the experiment. There are a few proposals and experimental implementations of twoqubit gates [2-7] with various levels of fidelity. In general, any two-qubit entangling gate can be used in the universal set of the quantum gates, hence entanglement generation is the crucial step in the two-qubit-gate realization. As a result, the creation and manipulation of the entangled states became an important research topic in quantum information processing [8] and quantum metrology [9].

During the last years, several methods have been proposed to create entanglement in quantum systems involving a small number of particles as well as multiparticle entanglement $[4,10,11]$. In general, we can divide the methods of entanglement state preparation into two distinct groups: the first one is based on an external field manipulation by the wave function of the subsystems while the interaction between subsystems is fixed; the second one is based on direct control of the interaction between the subsystems. The problem of creating and controlling entanglement is directly connected to the problem of coherent control of population transfer and can be mapped onto the problem of creating specific coherent superpositions in multilevel systems (assuming that the interaction is already included in the total system energylevel structure).

The standard quantum system underlying the two-qubit manipulation involves a four-level system in closed-loop configuration. When every state-to-state transition can be addressed independently, new forms of control are possible. In $[12,13]$, we have shown that the relative phase between the external fields controls population dynamics and can be used to generate entangled states due to the quantum interference between two pathways connecting the initial and target states [14]. It was shown that for properly chosen relative phases one can observe either Rabi oscillations according to the Mølmer-Sørensen (M-S) scheme [5-7] or collapse and revival phenomena, as in the well-known Jaynes-Cummings model $[15,16]$.

In this work we address the role of the relative phase between the pulses in the design of two-qubit quantum gates. Our paper is organized as follows. In Sec. II we setup general formalism and derive a general form of the evolution operator. In Sec. III we show decomposition of the CNOT quantum gate. Section IV is the conclusion.

\section{GENERAL EQUATIONS}

To address a general case, here we consider the dynamics of two distinguishable qubits in a one-dimensional harmonic trap assuming that the additional degrees of freedom are suppressed and neglecting decoherence effects. The collective motion of qubits is defined by an effective harmonic potential with the Hamiltonian

$$
H_{0}=\hbar \nu\left(\widehat{a}^{\dagger} \widehat{a}+\frac{1}{2}\right)+\sum_{i} \frac{E_{2}^{(i)}}{2}\left(\boldsymbol{I}-\boldsymbol{\sigma}_{z i}\right),
$$

where $v$ is the frequency of the vibrational motion, $E_{2}^{(i)}$ is the transition energy in the $i$ qubit (for instance, the excited internal state energy of the ion or atom), $\sigma_{z i}$ are Pauli matrices, and $\widehat{a}^{\dagger}, \widehat{a}$ are the vibrational ladder operators. The interaction of the qubits with the external fields can be written in the 
following form

$$
\begin{aligned}
& V_{\mathrm{int}}(t) \\
& \quad=-\hbar \sum_{j, i} \Omega_{j}(t) \cos \left[\omega_{j} t+\phi_{j}-\eta_{j}\left(\widehat{a}^{\dagger}+\widehat{a}\right)\right] \sigma_{x i}+\text { H.c. },
\end{aligned}
$$

where $\omega_{j}, \phi_{j}$ are the laser frequency and phase, $\Omega_{j}(t)$ is the Rabi frequency, $\eta_{j}=k_{j} \sqrt{\hbar / 4 m \omega_{t}}$ is the Lamb-Dicke parameter, $\omega_{t}$ is the trap frequency, $m$ is the ion mass, and $k_{j}$ is the laser wave vector.

The total two-qubit wave function has the form

$$
\begin{aligned}
|\Psi(t)\rangle= & a_{1}(t)|00 n\rangle+a_{2}(t)|10 n\rangle+a_{3}(t)|01 n\rangle+a_{4}(t)|11 n\rangle \\
& +b_{1}(t)|01 n-1\rangle+b_{2}(t)|10 n+1\rangle+b_{3}(t)|01 n+1\rangle \\
& +b_{4}(t)|10 n-1\rangle+c_{1}(t)|00 n-1\rangle+c_{2}(t)|00 n+1\rangle \\
& +c_{3}(t)|11 n-1\rangle+c_{4}(t)|11 n+1\rangle,
\end{aligned}
$$

which contains the computational subspace $(|00 n\rangle,|10 n\rangle,|01 n\rangle,|11 n\rangle)$ and additional ancillary states $(|00 n \pm 1\rangle,|10 n \pm 1\rangle,|01 n \pm 1\rangle,|11 n \pm 1\rangle) ; 0,1$ denote the internal states of the qubit, and $n, n \pm 1$ are the vibrational quantum numbers. Similar to the ingenious idea of the authors of [5-7], here we explore the excitation of the trapped ion by using off-resonant laser fields. The energy-level structure of the system and the corresponding coupling between states are shown in Fig. 1. To examine the general case, we use eight different fields with frequencies arranged in a pairwise way to accomplish two-photon resonance between the computational states while all ancillary states are not excited.

Assuming that all single-photon detunings $\delta_{1,2}=E_{2}^{1,2} / \hbar \mp$ $v-\omega_{1,2}$ and $\delta_{1,2}^{\prime}=E_{2}^{1,2} / \hbar \pm v-\omega_{1,2}^{\prime}$ are sufficiently large we make the adiabatic elimination of the ancillary vibrational states [17] and obtain the Schrödinger equation for the probability amplitudes of the computational states $a_{i}$. Obviously,

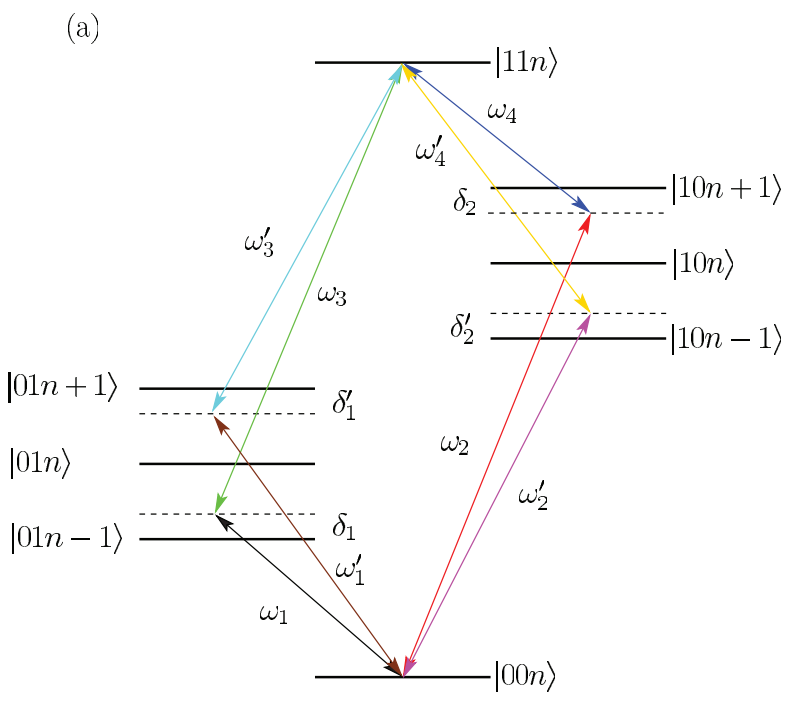

the general system of four differential equations splits for two uncoupled two-level systems. Since the expressions for the effective two-photon Rabi frequencies and ac Stark shifts are complicated we simplify the excitation scheme by choosing completely overlapped pulses for all external fields $\Omega_{j}(t)=$ $\Omega_{0}(t)$ and consider symmetric detunings $\delta_{1}=\delta_{2}^{\prime}, \delta_{2}=\delta_{1}^{\prime}$.

The effective Hamiltonian for states $|00 n\rangle$ and $|11 n\rangle$ [see Fig. 1(a)] is

$$
H_{\mathrm{eff}}=-\hbar\left(\begin{array}{cc}
\Omega_{\mathrm{ac}}(t) & \Omega_{e}(t) \\
\Omega_{e}^{*}(t) & \Omega_{\mathrm{ac}}(t)
\end{array}\right),
$$

where $\Omega_{\mathrm{ac}}(t)=\eta^{2} \Omega_{0}^{2}(t)\left[n / \delta_{1}+(n+1) / \delta_{2}\right] / 2$ is the ac Stark shift, and

$$
\begin{aligned}
\Omega_{e}(t)=\Omega_{14}(t)= & \eta^{2} \Omega_{0}^{2}(t)\left[n\left(e^{i \phi_{13}}+e^{i \phi_{24}^{\prime}}\right) / \delta_{1}\right. \\
& \left.+(n+1)\left(e^{i \phi_{24}}+e^{i \phi_{13}^{\prime}}\right) / \delta_{2}\right] / 4,
\end{aligned}
$$

is the effective two-photon Rabi frequency.

Correspondingly, the effective Hamiltonian for states $|01 n\rangle$ and $|10 n\rangle$ [see Fig. 1(b)] has the same form as in Eq. (4), with the ac Stark shift $\Omega_{\mathrm{ac}}(t)=\eta^{2} \Omega_{0}^{2}(t)\left[1 / \delta_{2}-1 / \delta_{1}\right] / 4$ and the effective two-photon Rabi frequency

$$
\begin{aligned}
\Omega_{e}(t)=\Omega_{23}(t)= & \eta^{2} \Omega_{0}^{2}(t)\left[\frac{n+1}{\delta_{2}} e^{i\left(\phi_{3}-\phi_{4}^{\prime}\right)}-\frac{n+1}{\delta_{1}} e^{i\left(\phi_{2}^{\prime}-\phi_{1}\right)}\right. \\
& \left.-\frac{n}{\delta_{2}} e^{i\left(\phi_{2}-\phi_{1}^{\prime}\right)}+\frac{n}{\delta_{1}} e^{i\left(\phi_{3}^{\prime}-\phi_{4}\right)}\right] / 4
\end{aligned}
$$

As we observe, the ac Stark shift and the effective two-photon Rabi frequency depend (in the general case) on the vibrational quantum number. The Hamiltonian is a bit more simplified if we symmetrize the detunings by taking $\delta_{2}=-\delta_{1}=\delta_{0}$. This condition effectively addresses the case of two indistinguishable ions excited by only two external fields and we need to impose the following relations for the phases $\phi_{1} \equiv \phi_{2}^{\prime} \equiv \phi_{4} \equiv \phi_{3}^{\prime}$ and $\phi_{2} \equiv \phi_{1}^{\prime} \equiv \phi_{3} \equiv \phi_{4}^{\prime}$. Under

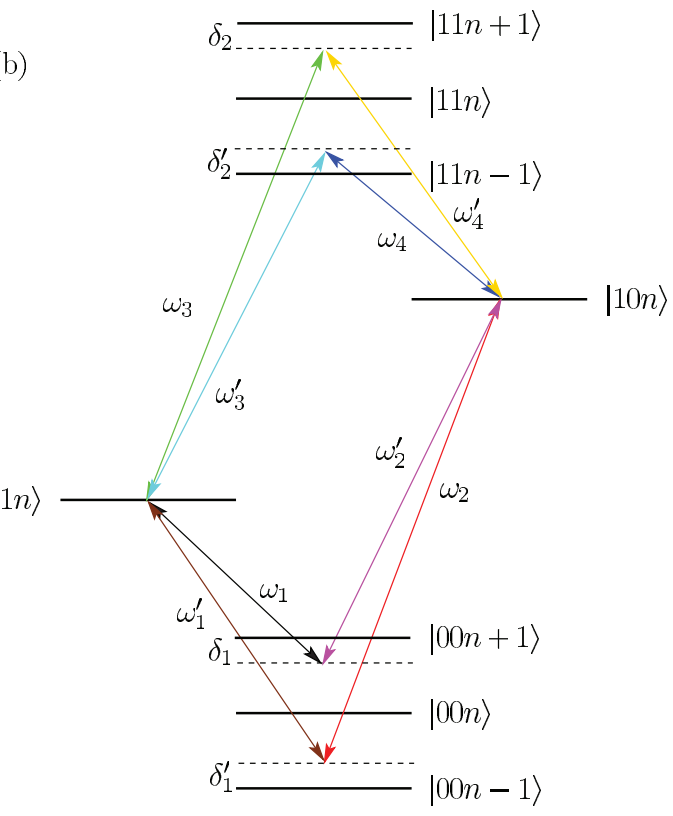

FIG. 1. (Color online) Energy-level structure of the two-ion system and corresponding coupling by external fields. To simplify the figure, we show the couplings between the $|00 n\rangle,|11 n\rangle$ states, and between the $|01 n\rangle,|10 n\rangle$ states in two frames, (a) and (b), respectively. 
these conditions the ac shifts for all computational states are identical, $\Omega_{\mathrm{ac}}(t)=\eta^{2} \Omega_{0}^{2}(t) /\left(2 \delta_{0}\right)$, while the Rabi frequencies are

$$
\Omega_{14}(t)=\eta^{2} \Omega_{0}^{2}(t) e^{i\left(\phi_{1}+\phi_{2}\right)} /\left(2 \delta_{0}\right), \quad \Omega_{23}(t)=\eta^{2} \Omega_{0}^{2}(t) /\left(2 \delta_{0}\right) .
$$

The independence of the Rabi frequencies on the vibrational quantum number shows explicitly that the considered excitation scheme does not require laser cooling to the motional ground state [5].

The ac Stark shift is irrelevant for the dynamics of the two-qubit system since it produces only the dynamic global phase defined by $\zeta(t)=\int_{0}^{t} d t^{\prime} \Omega_{\mathrm{ac}}\left(t^{\prime}\right)$ that can be excluded from the Hamiltonian by the unitary transformation $a_{i}(t)=$ $\bar{a}_{i}(t) e^{i \zeta(t)}$. Therefore, the total Hamiltonian in the limit of adiabatic elimination of the ancillary states can be taken as

$$
\begin{aligned}
H_{T}(t) & =-\hbar \eta^{2} \Omega_{0}^{2}(t) /\left(2 \delta_{0}\right)\left[\cos \left(\phi_{+} / 2\right) \sigma_{x}-\sin \left(\phi_{+} / 2\right) \sigma_{y}\right] \otimes\left[\cos \left(\phi_{+} / 2\right) \sigma_{x}-\sin \left(\phi_{+} / 2\right) \sigma_{y}\right] \\
& =-\hbar \eta^{2} \Omega_{0}^{2}(t) /\left(2 \delta_{0}\right)\left(\begin{array}{cc}
0 & e^{i \phi_{+} / 2} \\
e^{-i \phi_{+} / 2} & 0
\end{array}\right) \otimes\left(\begin{array}{cc}
0 & e^{i \phi_{+} / 2} \\
e^{-i \phi_{+} / 2} & 0
\end{array}\right)
\end{aligned}
$$

where $\phi_{+}=\phi_{1}+\phi_{2}$, and $\sigma_{x, y}$ are the Pauli matrices.

Solving the Schrödinger equation with the Hamiltonian in Eq. (8), the complete evolution operator [up to the global phase $\zeta(t)]$ in the computational basis $\{|00\rangle,|01\rangle,|10\rangle,|11\rangle\}$ can be written as

$$
\begin{aligned}
& U_{t}(\xi)=\exp \left[-\frac{i}{\hbar} \int_{0}^{t} H_{T}\left(t^{\prime}\right) d t^{\prime}\right]=\exp \left\{i \xi(t)\left[\cos \left(\phi_{+} / 2\right) \sigma_{x}-\sin \left(\phi_{+} / 2\right) \sigma_{y}\right] \otimes\left[\cos \left(\phi_{+} / 2\right) \sigma_{x}-\sin \left(\phi_{+} / 2\right) \sigma_{y}\right]\right\} \\
& =\left(\begin{array}{cccc}
\cos \xi(t) & 0 & 0 & i e^{i \phi_{+}} \sin \xi(t) \\
0 & \cos \xi(t) & i \sin \xi(t) & 0 \\
0 & i \sin \xi(t) & \cos \xi(t) & 0 \\
i e^{-i \phi_{+}} \sin \xi(t) & 0 & 0 & \cos \xi(t)
\end{array}\right),
\end{aligned}
$$

where $\xi(t)=\eta^{2} \int_{0}^{t} d t^{\prime} \Omega_{0}^{2}\left(t^{\prime}\right) /\left(2 \delta_{0}\right)$ is the effective pulse area. The global phase factor resulting from the ac Stark shift can be taken into account multiplying $U_{t}(\xi)$ in Eq. (9) by $e^{i \xi(t) \boldsymbol{I} \otimes \boldsymbol{I}}$, where $\boldsymbol{I}$ is the $2 \times 2$ identity matrix.

After some algebra, we find the general canonical form of the evolution operator in Eq. (9) as

$$
U_{t}(\xi)=C_{1} \otimes C_{2} e^{i \xi(t) \sigma_{x} \otimes \sigma_{x}} C_{1}^{-1} \otimes C_{2}^{-1}=C_{1} \otimes C_{2}\left(\begin{array}{cccc}
\cos \xi(t) & 0 & 0 & i \sin \xi(t) \\
0 & \cos \xi(t) & i \sin \xi(t) & 0 \\
0 & i \sin \xi(t) & \cos \xi(t) & 0 \\
i \sin \xi(t) & 0 & 0 & \cos \xi(t)
\end{array}\right) C_{1}^{-1} \otimes C_{2}^{-1}
$$

where $C_{1,2}=e^{i \phi_{+} \sigma_{z} / 4}$.

\section{CNOT GATE DECOMPOSITION}

The canonical decomposition of $U_{t}(\xi)$, Eq. (10), means that the phase factors $e^{ \pm i \phi_{+}}$in Eq. (9) can always be taken into account by applying the single-qubit phase gates defined by $C_{1,2}$. Therefore, we can use the evolution operator $U_{x x}(\xi)=$ $\exp \left[i \xi(t) \sigma_{x} \otimes \sigma_{x}\right]$ to design for example a CNOT gate, taking into account the phase factors $e^{ \pm i \phi_{+}}$at the end by adding the corresponding local single-qubit phase gates $C_{1,2}$. With the effective pulse area $\xi(T)=\pi / 4$, where $T$ indicates the end of the pulses, we obtain

$$
\begin{aligned}
U_{\mathrm{CNOT}} & =e^{-i \pi / 4} B_{1} \otimes \boldsymbol{I} U_{x x}\left(\frac{\pi}{4}\right) A_{1} \otimes A_{2} \\
& =e^{-i \pi / 4} B_{1} \otimes \boldsymbol{I} e^{i \frac{\pi}{4} \sigma_{x} \otimes \sigma_{x}} A_{1} \otimes A_{2} \\
& =e^{-i \pi / 4} \frac{1}{\sqrt{2}} B_{1} \otimes \boldsymbol{I}\left(\begin{array}{cccc}
1 & 0 & 0 & i \\
0 & 1 & i & 0 \\
0 & i & 1 & 0 \\
i & 0 & 0 & 1
\end{array}\right) A_{1} \otimes A_{2}
\end{aligned}
$$

where

$$
\begin{aligned}
& B_{1}=\frac{i}{\sqrt{2}}\left(\begin{array}{cc}
-1 & 1 \\
1 & 1
\end{array}\right) \quad A_{1}=\frac{1}{2}\left(\begin{array}{cc}
1-i & 1+i \\
-1+i & 1+i
\end{array}\right) \\
& A_{2}=-\frac{1}{\sqrt{2}}\left(\begin{array}{ll}
1 & i \\
i & 1
\end{array}\right) .
\end{aligned}
$$

Taking into consideration Eq. (10) we have

$U_{\mathrm{CNOT}}=e^{-i \pi / 4} B_{1} \otimes I C_{1}^{-1} \otimes C_{2}^{-1} U_{t}\left(\frac{\pi}{4}\right) C_{1} \otimes C_{2} A_{1} \otimes A_{2}$.

Note, the decomposition of Eq. (13) is valid for an arbitrary phase $\phi_{+}$.

It is worth pointing out that the canonical form of $U_{t}(\xi)$ in Eq. (10) is equivalent to the Cartan decomposition [18] of an element of the group $\mathrm{SU}(4)$. This is of the form $U=k_{1} U_{A} k_{2}$, where $k_{1}$ and $k_{2}$ represent single-qubit operations from the $\mathrm{SU}(2) \otimes \mathrm{SU}(2)$ subgroup of SU(4). They are given explicitly 
in our case as

$$
\begin{gathered}
k_{1}=C_{1} \otimes C_{2}=e^{i\left(\boldsymbol{\sigma}_{z} \otimes \boldsymbol{I}+\boldsymbol{I} \otimes \boldsymbol{\sigma}_{z}\right) \phi_{+} / 4}, \\
k_{2}=C_{1}^{-1} \otimes C_{2}^{-1}=e^{-i\left(\boldsymbol{\sigma}_{z} \otimes \boldsymbol{I}+\boldsymbol{I} \otimes \boldsymbol{\sigma}_{z}\right) \phi_{+} / 4} .
\end{gathered}
$$

In general, the central part of the Cartan decomposition $U=k_{1} U_{A} k_{2}$ is generated by elements of the maximal Abelian subalgebra of the algebra $\mathrm{SU}(4)$, which is spanned by the set of three commuting generators, usually chosen as $\left\{\sigma_{x} \otimes\right.$ $\left.\boldsymbol{\sigma}_{x}, \boldsymbol{\sigma}_{y} \otimes \boldsymbol{\sigma}_{y}, \boldsymbol{\sigma}_{z} \otimes \boldsymbol{\sigma}_{z}\right\}$. These are nonlocal generators responsible for the entangling capabilities of a unitary operation $U$.

In our specific case, the Cartan decomposition of $U_{t}(\xi)$ is given as

$$
U_{t}(\xi)=k_{1}\left(\phi_{+}\right) U_{x x}(\xi) k_{2}\left(\phi_{+}\right),
$$

which explicitly underlines the fact that the entangling capabilities of $U_{t}(\xi)$, contained in $U_{x x}(\xi)$, are invariant to any variations of the phase $\phi_{+}$including phase errors caused, for example, by imperfections or physical constraints of a given implementation. This makes the gate naturally more robust to phase errors. Moreover, as pointed above it allows to correct these errors by single-qubit operations that are usually easier to implement.

The Cartan decomposition of $U_{t}(\xi)$ illuminates another aspect of this gate, specifically that it can be extended to a large family of unitary operations of the same entangling content, or in other words, belonging to the same local equivalence class $[18,19]$. These may be more convenient to work with for conceptual or implementation reasons. Specifically, any unitary operation generated by the maximal Abelian subalgebra of SU(4), $U_{A}=\exp \left[i\left(c_{x} \boldsymbol{\sigma}_{x} \otimes \boldsymbol{\sigma}_{x}+c_{y} \boldsymbol{\sigma}_{y} \otimes \boldsymbol{\sigma}_{y}+c_{z} \boldsymbol{\sigma}_{z} \otimes\right.\right.$ $\left.\sigma_{z}\right)$ ], commutes with $U_{x x}(\xi)$, thus allowing us to construct the family of locally equivalent gates $U_{t}^{\prime}(\xi)$ defined as

$$
\begin{aligned}
U_{t}^{\prime}(\xi) & =\left[k_{1}\left(\phi_{+}\right) U_{A}\right] U_{x x}(\xi)\left[U_{A}^{-1} k_{2}\left(\phi_{+}\right)\right] \\
& =\left[\left(C_{1} \otimes C_{2}\right) U_{A}\right] U_{x x}(\xi)\left[U_{A}^{-1}\left(C_{1}^{-1} \otimes C_{2}^{-1}\right)\right] .
\end{aligned}
$$

To demonstrate the usefulness of this relation, showing local equivalence of the gates, we consider an alternative way to obtain the complete evolution operator in Eq. (9). We can take care of the phase factors $e^{ \pm i \phi_{+}}$in Eq. (9) by using the following transformation

$$
U_{\phi}=\left(\begin{array}{cccc}
e^{i \phi_{+}} & 0 & 0 & 0 \\
0 & 1 & 0 & 0 \\
0 & 0 & 1 & 0 \\
0 & 0 & 0 & 1
\end{array}\right)
$$

In this way, the complete evolution operator can be written in the form

$$
U_{t}(\xi)=U_{\phi} e^{i \xi(t) \sigma_{x} \otimes \sigma_{x}} U_{\phi}^{-1}=U_{\phi} U_{x x}(\xi) U_{\phi}^{-1} .
$$

It can be shown exactly that Eq. (19) is equivalent to the decomposition Eq. (10) as

$$
\begin{aligned}
U_{t}(\xi) & =U_{\phi} e^{i \xi(t) \sigma_{x} \otimes \sigma_{x}} U_{\phi}^{-1}=U_{\phi} U_{x x}(\xi) U_{\phi}^{-1} \\
& =\left(C_{1} \otimes C_{2}\right) U_{z z}\left(\phi_{+} / 4\right) U_{x x}(\xi) U_{z z}\left(-\phi_{+} / 4\right)\left(C_{1}^{-1} \otimes C_{2}^{-1}\right) \\
& =\left(C_{1} \otimes C_{2}\right) U_{x x}(\xi)\left(C_{1}^{-1} \otimes C_{2}^{-1}\right),
\end{aligned}
$$

where we have canceled the irrelevant global phase factors. The difference between Eqs. (10) and (19) is in the physical implementation of these two decompositions; the point is that $U_{\phi}$ is the two-qubit phase (not local) gate while $C_{1} \otimes C_{2}$ are two local single-qubit gates, which do not contain any entanglement or nonlocality.

\section{CONCLUSION}

We presented a general canonical decomposition of the evolution operator of a two-qubit system in the one-dimensional harmonic trap. The relative phase between external fields is taken into account explicitly. The general form of the evolution operator shows directly the importance of the relative phase in designing two-qubit gate. As an example, we specifically focused on the excitation scheme that utilizes two laser pulses, and obtained the exact form of the two-qubit controlled-NOT gate. Based on the canonical decomposition of the evolution operator we demonstrated that the relative phase errors can be successfully corrected locally by applying appropriate singlequbit phase gates. Moreover, we argue that applying identical fields (for example, using a single field source and a set of beam splitters) to implement both the two-qubit entangling gate [consider $U_{x x}(\xi)$ as an example] and single-qubit gates $\left(C_{1,2}\right)$, makes the CNOT gate independent of the relative phase. Therefore, the CNOT gate becomes immune to the phase errors related to the phase fluctuations of the external fields. The presented results can facilitate further advances in the practical implementation of the circuit-based quantum computation.

\section{ACKNOWLEDGMENTS}

The authors thank Klaus Mølmer for valuable discussions. This research was supported in part by the National Science Foundation under Grant No. PHY11-25915. I.S.R. acknowledges financial support from the MICINN Project CTQ2012-36184. J.V. acknowledges the financial support of Science Foundation Ireland under Principal Investigator Award No. 10/IN.1/I3013.
[1] A. Barenco, C. H. Bennett, R. Cleve, D.P. DiVincenzo, N. Margolus, P. Shor, T. Sleator, J. A. Smolin, and H. Weinfurter, Phys. Rev. A 52, 3457 (1995).

[2] T. Sleator and H. Weinfurter, Phys. Rev. Lett. 74, 4087 (1995).

[3] J. I. Cirac and P. Zoller, Nature (London) 404, 579 (2000).
[4] F. Schmidt-Kaler et al., Nature (London) 422, 408 (2003).

[5] K. Mølmer and A. Sørensen, Phys. Rev. Lett. 82, 1835 (1999).

[6] A. Sørensen and K. Mølmer, Phys. Rev. Lett. 82, 1971 (1999).

[7] A. Sørensen and K. Mølmer, Phys. Rev. A 62, 022311 (2000). 
[8] H. Häffner, C. F. Roos, and R. Blatt, Phys. Rep. 469, 155 (2008).

[9] R. Horodecki, P. Horodecki, M. Horodecki, and K. Horodecki, Rev. Mod. Phys. 81, 865 (2009).

[10] D. Leibfried et al., Nature (London) 422, 412 (2003).

[11] T. D. Ladd, F. Jelezko, R. Laflamme, Y. Nakamura, C. Monroe and J. L. O'Brien, Nature (London) 464, 45 (2010).

[12] V. S. Malinovsky and I. R. Solá, Phys. Rev. Lett. 93, 190502 (2004); Phys. Rev. A 70, 042304 (2004); 70, 042305 (2004).

[13] V. S. Malinovsky and I. R. Solá, Quantum Inf. Comput. 5, 364 (2005).
[14] P. W. Brumer and M. Shapiro, Principles of the Quantum Control of Molecular Processes (Wiley \& Sons, Hoboken, NJ, 2003).

[15] E. T. Jaynes and F. W. Cummings, Proc. IEEE 51, 89 (1963); M. Tavis and F. W. Cummings, Phys. Rev. 170, 379 (1968).

[16] B. W. Shore and P. L. Knight, J. Mod. Opt. 40, 1195 (1993).

[17] P. R. Berman and V. S. Malinovsky, Principles of Laser Spectroscopy and Quantum Optics (Princeton University Press, Princeton, NJ, 2011).

[18] J. Zhang, J. Vala, S. Sastry, and K. B. Whaley, Phys. Rev. A 67, 042313 (2003).

[19] Y. Makhlin, Quantum Inf. Proc. 1, 243 (2002). 